\title{
Effects of the symmetry energy on the kaon condensates in the quark-meson coupling model
}

\author{
Prafulla K. Panda, ${ }^{1,2,3}$ Débora P. Menezes, ${ }^{3,4}$ and Constança Providência ${ }^{2}$ \\ ${ }^{1}$ Department of Physics, C.V. Raman College of Engineering, Vidya Nagar, Bhubaneswar 752054, India \\ ${ }^{2}$ Centro de Física Computacional, Department of Physics, University of Coimbra, P-3004-516 Coimbra, Portugal \\ ${ }^{3}$ Departamento de Física, CFM, Universidade Federal de Santa Catarina, C.P. 476, CEP 88.040-900 Florianópolis, Santa Catarina, Brazil \\ ${ }^{4}$ Departamento de Física Aplicada, Universidad de Alicante, Apartado de Correus 99, E-03080, Alicante, Spain
}

(Received 12 November 2013; revised manuscript received 18 March 2014; published 23 April 2014)

\begin{abstract}
In this work we investigate protoneutron star properties within a modified version of the quark-meson coupling (QMC) model that incorporates an $\omega-\rho$ interaction plus kaon condensed matter at finite temperature. Fixed entropy and trapped neutrinos are taken into account. Our results are compared with the ones obtained with the GM1 parametrization of the nonlinear Walecka model for similar values of the symmetry energy slope. Contrary to GM1, within the QMC model the formation of low mass black holes during cooling are not probable. It is shown that the evolution of the protoneutron star may include the melting of the kaon condensate driven by the neutrino diffusion, followed by the formation of a second condensate after cooling. The signature of this complex process could be a neutrino signal followed by a gamma ray burst. We have seen that both models can, in general, describe very massive stars.
\end{abstract}

DOI: 10.1103/PhysRevC.89.045803

PACS number(s): 26.60.-c, 24.10.Jv, 21.65.-f, 95.30.Tg

\section{INTRODUCTION}

In recent years, all sorts of phenomenological equations of state $(\mathrm{EoS})$, relativistic and nonrelativistic ones, have been used to describe (proto)neutron star matter. These EoS are parameter dependent and are adjusted so as to reproduce nuclear matter bulk properties, such as the binding energy at the correct saturation density and incompressibility as well as ground state properties of some nuclei and their collective responses [1,2]. Attempts to constrain the EoS have been made and they were based either on finite nuclei experimental results-for instance, the isoscalar monopole and the isovector dipole giant resonances [3] and neutron skin thickness [4] - or on astrophysical observations [5,6]. Until 2010 , when a star with a mass of almost $2 M_{\odot}$ was confirmed [7], most EoS were expected to produce maximum stellar masses just larger than $1.44 M_{\odot}$ and radii of the order of 10 to $12 \mathrm{~km}$. Some of them, such as the NL3 parametrization [2] of the nonlinear Walecka model (NLWM) were even discarded, considered to be too hard and to provide too large solar masses. Recently a second very massive neutron star was detected [8] and many parametrizations and models were revisited and readjusted to account for the new observations. Also, many other constraints based on the above mentioned nuclear properties and also on the symmetry energy, its slope, skewness, dipole polarizabilities, heavy-ion collision flows, isobaric analog states, etc., have been proposed $[9,10]$.

As far as relativistic models are concerned, the $\omega-\rho$ interaction $[11,12]$ can be adjusted to reproduce experimental values of the symmetry energy and its related slope, the latest being strongly correlated with many nuclear [13] and stellar properties [14].

On the other hand, it is well known that the EoS and the internal constitution of the neutron stars depend on the nature of the strong interaction. In a compact star, strangeness may occur in the form of baryons, such as $\Lambda$ and $\Sigma^{-}$hyperons, as a Bose condensate, i.e., $K^{-}$meson condensate, or in the form of strange quarks, in all cases influencing the star structure and its macroscopic properties [15,16]. Some years ago, it was suggested that, above some critical density, the ground state of baryonic matter might contain a Bose-Einstein condensate of negatively charged kaons [17] if a pion condensate also exists. In [18], another mechanism that allowed kaon condensation without pion condensation was proposed for the first time. There is a strong attraction between $K^{-}$mesons and baryons which increases with density and lowers the energy of the zero momentum state. A condensate is formed when this energy equals the kaon chemical potential $\mu$. When the electron chemical potential equals the effective kaon mass, the kaons are favored because they help in the conservation of charge neutrality once they are bosons and can condense in the lowest energy state. For this reason $K^{-}$mesons that have the same electric charge as electrons are the type of kaons that normally appear in a condensed state in stars.

At this point, it is important to bear in mind that the presence of hyperons strongly influences the possible onset of (anti)kaons, as discussed in $[19,20]$ for zero temperature systems. However, as hyperonic stellar matter has already been extensively discussed within the quark-meson coupling (QMC) model [21-23], in this work we restrict ourselves to the possible appearance of the kaon condensation in finite temperature systems. It is well known that the onset of the kaon condensation is model dependent and varies according to the strength of the kaon optical potential [20,24,25]. In the present work, we revisit the possibility that a hybrid compact star can be constituted by hadrons and kaon condensed matter at higher densities [20] by using the quark-meson coupling model at finite temperature $[21,26]$ with the inclusion of the $\omega-\rho$ interaction $[11,12]$. The inclusion of this nonlinear interaction was shown to soften the symmetry energy at high densities and to bring the QMC model properties closer to density dependent relativistic models [22]. As the inclusion of this term allows us to tune the slope of the symmetry energy, shown to be strongly correlated with some star properties, it is an important ingredient in the investigation that follows. In 
a previous work [27] a discussion on the onset of kaons and antikaons controlled by stiff and soft symmetry energy and EoS was performed at zero temperature with four kinds of models: a standard relativistic mean-field one, a density dependent model, a model with the $\omega$ - $\rho$ interaction, and a model with higher order coupling constants. It was found that, although the last two models bear quite different symmetry energies, they yield very similar star masses and radii. In [28], it was seen that the effects of kaon condensation on metastable stars can be quite dramatic, resulting in different neutrino emission signals. Hence, for a better understanding of the role played by kaons inside a star, we use two different models, the QMC and the GM1 [16] parametrization of the NLWM for three values of fixed entropies that correspond to different snapshots of the star evolution and discuss the effect of the symmetry energy. The properties of the stars are also studied.

In the QMC model, nucleons are described as a system of nonoverlapping bags that interact through the scalar and vector mesons. The quark degrees of freedom are explicitly taken into account and the couplings are determined at the quark level. In the present work we also treat the kaons as MIT bags [29], and the couplings of the kaons with nucleons are determined in a self-consistent way.

In Sec. II, we discuss the formalism employed in finite temperature calculations. In Sec. III we present and discuss our results and compare them with the GM1 parametrization of the nonlinear Walecka model [16]. For this comparison, in view of the fact that the symmetry energy slope regulates some physical properties, it was chosen to be similar in both models. In the final section, we draw our conclusions.

\section{FORMALISM}

\section{A. QMC equation of state and the symmetry energy}

In the QMC model, the nucleon in nuclear medium is assumed to be a static spherical MIT bag in which quarks interact with the scalar $(\sigma)$ and vector $(\omega, \rho)$ fields, and those are treated as classical fields in the mean-field approximation (MFA). The quark $\psi_{q}(\vec{r}, t)$ inside the bag satisfies the equation of motion

$$
\begin{aligned}
& {\left[i \gamma_{\mu} \partial^{\mu}-\left(m_{q}^{0}-g_{\sigma}^{q} \sigma_{0}\right)-\gamma^{0}\left(g_{\omega}^{q} \omega_{0}+\frac{1}{2} g_{\rho}^{q} \tau_{3 q} b_{03}\right)\right] \psi_{q}(\vec{r}, t)} \\
& \quad=0
\end{aligned}
$$

where $\sigma_{0}, \omega_{0}, b_{03}$ are the classical meson fields for $\sigma, \omega$, $\rho$ mesons, $m^{0}{ }_{q}$ is the current quark mass, and $\tau_{3 q}$ is the third component of the Pauli matrices. $g_{\sigma}^{q}, g_{\omega}^{q}, g_{\rho}^{q}$ denote the quark coupling constants with $\sigma, \omega, \rho$. At finite temperature, quarks inside the bag can be thermally excited to higher angular momentum states and also quark antiquark pairs can be created. For simplicity, the bag is assumed to be spherical with radius $R$ which depends on the temperature. The single-particle energies in units of $R^{-1}$ for the quarks and the antiquarks are given as

$$
\epsilon_{q}^{n k}=\Omega_{q}^{n k}+R_{N}\left(V_{\omega} \pm \frac{1}{2} V_{\rho}\right)
$$

and

$$
\epsilon_{\bar{q}}^{n k}=\Omega_{q}^{n k}-R_{N}\left(V_{\omega} \pm \frac{1}{2} V_{\rho}\right)
$$

where $V_{\sigma}=g_{\sigma}^{q} \sigma_{0}, V_{\omega}=g_{\omega}^{q} \omega_{0}$ and $V_{\rho}=g_{\rho}^{q} b_{03}$. The plus sign refers to the $u$ quarks and the minus sign to the $d$ quarks. The total energy from the quarks and antiquarks at finite temperature is

$$
E_{\mathrm{tot}}=\sum_{q, n, k} \frac{\Omega_{q}^{n k}}{R_{N}}\left(f_{n k}^{q}+f_{n k}^{\bar{q}}\right),
$$

where

$$
f_{n k}^{q}=\frac{1}{e^{\left[\Omega_{q}^{n k} / R_{N}-v_{q}\right] / T}+1}
$$

and

$$
f_{n k}^{\bar{q}}=\frac{1}{e^{\left[\Omega_{q}^{n k} / R_{N}+v_{q}\right] / T}+1},
$$

with $\Omega_{q}^{n k}=\sqrt{x_{n k}^{2}+R_{N}^{2} m_{q}^{* 2}}, m_{q}^{*}=m_{q}^{0}-V_{\sigma}$, and the eigenvalues $x_{n k}$ for the state characterized by $n$ and $k$ are determined by the boundary condition at the bag surface. In the above, $v_{q}=\mu_{q}-V_{\omega}-m_{\tau}^{q} V_{\rho}$ is the effective quark chemical potential and is related to the quark chemical potential, $\mu_{q}$. The energy of a static baryon bag consisting of three ground state quarks can be expressed as

$$
E_{N}^{\mathrm{bag}}=E_{\mathrm{tot}}-\frac{Z_{N}}{R_{N}}+\frac{4}{3} \pi R_{N}^{3} B_{N},
$$

where $Z_{N}$ is the parameter that accounts for zero point motion and $B_{N}$ is the bag constant. The entropy of the bag is defined as

$$
\begin{aligned}
\mathcal{S}^{\mathrm{bag}}= & -\sum_{q, n, k}\left[f_{n k}^{q} \ln f_{n k}^{q}+\left(1-f_{n k}^{q}\right) \ln \left(1-f_{n k}^{q}\right)\right. \\
& \left.+\bar{f}_{n k}^{q} \ln \bar{f}_{n k}^{q}+\left(1-\bar{f}_{n k}^{q}\right) \ln \left(1-\bar{f}_{n k}^{q}\right)\right] .
\end{aligned}
$$

The free energy of the bag is given by $F_{N}^{\text {bag }}=E_{N}^{\text {bag }}-T \mathcal{S}^{\text {bag }}$ and the effective mass of a nucleon bag at rest is taken to be $M_{N}^{*}=F_{N}^{\text {bag }}$. The equilibrium condition for the bag is obtained by minimizing the effective mass $M_{N}^{*}$ with respect to the bag radius $R_{N}$,

$$
\frac{\partial M_{N}^{*}}{\partial R_{N}^{*}}=0
$$

Once the bag radius is obtained, the effective baryon mass is immediately determined. For a given temperature and scalar field $\sigma$, the effective quark chemical potentials $v_{q}$ are determined from the total number of quarks, isospin density, and strangeness.

We next obtain the thermodynamic potential within the mean-field approximation and perform a calculation similar to that carried out in [20]. We assume that the kaons are described by the static MIT bag in the same way as the nucleons. Moreover $\sigma, \omega$, and $\rho$ mesons are only mediators of the $u$ and $d$ quarks inside the kaons. The effective Lagrangian density for the kaon sector is

$$
\mathcal{L}_{K}=D_{\mu}^{*} K^{*} D^{\mu} K-M_{K}^{* 2} K^{*} K
$$

where kaons are coupled to the meson fields via minimal coupling and the covariant derivative reads

$$
D_{\mu}=\partial_{\mu}+i X_{\mu},
$$


with

$$
X_{\mu}=g_{\omega K} \omega_{\mu}+\frac{g_{\rho K}}{2} \vec{\tau} \cdot \vec{b}_{\mu} .
$$

The energy of the static bag describing kaon $\mathrm{K}$ can be expressed as

$$
E_{K}^{\mathrm{bag}}=\sum_{q, n, k} \frac{\Omega_{q}^{n k}}{R_{K}}\left(f_{n k}^{q}+f_{n k}^{\bar{q}}\right)-\frac{Z_{K}}{R_{K}}+\frac{4}{3} \pi R_{K}^{3} B_{K} .
$$

For our calculations, we have fixed the bag constant, $B_{K}$, to be the same as for the nucleon, and from the kaon mass and the stability condition in the vacuum we have obtained $Z_{K}=$ 3.362 and $R_{K}=0.457 \mathrm{fm}$ for $R_{N}=0.6 \mathrm{fm}$. The free energy of the kaon bag is $F_{K}^{\mathrm{bag}}=E_{K}^{\mathrm{bag}}-T \mathcal{S}^{\mathrm{bag}}$ and the effective mass of a kaon bag at rest is taken to be $M_{K}^{*}=F_{K}^{\text {bag }}$. In analogy with the nucleonic sector, the equilibrium condition for the bag is

$$
\frac{\partial M_{K}^{*}}{\partial R_{K}^{*}}=0 .
$$

The Bose occupation probability for particles $\left(f_{B^{+}}\right)$and antiparticles $\left(f_{B^{-}}\right)$appears naturally in the equation of state and reads

$$
f_{B^{ \pm}}=\frac{1}{\left(e^{\beta\left(\omega^{ \pm} \mp v_{K}\right)}-1\right)}
$$

with $\beta=1 / T, \epsilon_{K}^{*}=\sqrt{p^{2}+M_{K}^{* 2}}$ and $\omega^{ \pm}=\epsilon_{K}^{*} \pm X_{0}$. In the above we define the kaon effective chemical potential $v_{K}=$ $\mu_{K}+X_{0}$ where $X_{0}=g_{\omega K} \omega_{0}+g_{\rho K} b_{03} / 2$.

In the mean-field approximation, the kaon contribution to the thermodynamic potential is

$$
\begin{aligned}
\frac{\Omega_{K}}{V}= & \zeta^{2}\left[M_{K}^{* 2}-\left(\mu_{K}+X_{0}\right)^{2}\right] \\
& +T \int_{0}^{\infty} \frac{d^{3} p}{(2 \pi)^{3}}\left\{\ln \left[1-e^{-\beta\left(\omega^{-}+\mu\right)}\right]+\ln \left[1-e^{-\beta\left(\omega^{+}-\mu\right)}\right]\right\},
\end{aligned}
$$

from which we get

$$
\begin{aligned}
P_{K}= & -\frac{\Omega_{K}}{V}=\zeta^{2}\left[\left(\mu_{K}+X_{0}\right)^{2}-M_{K}^{* 2}\right] \\
& +\frac{1}{3} \int_{0}^{\infty} \frac{d^{3} p}{(2 \pi)^{3}} \frac{p^{2}}{\epsilon_{K}^{*}}\left[f_{B^{+}}+f_{B^{-}}\right]
\end{aligned}
$$

and the kaon contribution to the energy density reads

$$
\begin{aligned}
\varepsilon_{K}= & \zeta^{2}\left[M_{K}^{* 2}+\left(\mu_{K}^{2}+X_{0}^{2}\right)\right] \\
& +\int_{0}^{\infty} \frac{d^{3} p}{(2 \pi)^{3}}\left[\omega^{+}(p) f_{B^{+}}+\omega^{-}(p) f_{B^{-}}\right] \\
\equiv & \zeta^{2}\left[M_{K}^{* 2}+\left(\mu_{K}^{2}+X_{0}^{2}\right)\right]+\int_{0}^{\infty} \frac{d^{3} p}{(2 \pi)^{3}} \epsilon_{K}^{*}\left[f_{B^{+}}+f_{B^{-}}\right]
\end{aligned}
$$

The kaon number density is

$$
n_{K}=n_{K}^{c}+n_{K}^{t h}
$$

where $n_{K}^{c}=2 \zeta^{2}\left(\mu_{K}+X_{0}\right)$ is the condensate contribution and $n_{K}^{\text {th }}$ is the thermal contribution for the number density given by

$$
n_{K}^{t h}=\int_{0}^{\infty} \frac{d^{3} p}{(2 \pi)^{3}}\left[f_{B^{+}}-f_{B^{-}}\right] .
$$

Similarly the scalar density for the kaons is given by

$$
n_{K}^{s}=\int_{0}^{\infty} \frac{d^{3} p}{(2 \pi)^{3}} \frac{M_{K}^{*}}{\epsilon_{K}^{*}}\left[f_{B^{+}}+f_{B^{-}}\right]
$$

The kaon entropy density is given by $s_{K}=\beta\left(\varepsilon_{K}+P_{K}-\right.$ $\left.\mu_{K} n_{K}\right)$. [23]

The equations of motion for the meson fields are given by

$$
\begin{aligned}
m_{\sigma}^{2} \sigma= & \sum_{i=p, n}-\frac{\partial M_{N}^{*}}{\partial \sigma} \\
& \times \frac{1}{\pi^{2}} \int d \mathbf{k} \frac{M_{N}^{*}}{\left[k^{2}+M_{N}^{* 2}\right]^{1 / 2}}\left(f_{i}+\bar{f}_{i}\right), \\
& +g_{\sigma K}\left(n_{K}^{c}+n_{K}^{s}\right), \\
m_{\omega}{ }^{2} \omega_{0}= & \sum_{i=p, n} g_{\omega} \rho_{i}-g_{\omega K} n_{K}-2 \Lambda_{v} g_{\omega}^{2} g_{\rho}^{2} b_{03}^{2} \omega_{0}, \\
m_{\rho}{ }^{2} b_{03}= & \sum_{i=p, n} g_{\rho} I_{3 i} \rho_{i}-\frac{1}{2} g_{\rho K} n_{K}-2 \Lambda_{v} g_{\omega}^{2} g_{\rho}^{2} b_{03} \omega_{0}^{2},
\end{aligned}
$$

and

$$
\zeta\left[\mu_{k}-\omega^{+}(0)\right]\left[\mu_{k}+\omega^{-}(0)\right]=0,
$$

where $f_{i}$ and $\bar{f}_{i}$ are the thermal distribution functions for the baryon and antibaryon:

$$
f_{i}=\frac{1}{e^{\left(\epsilon^{*}-v\right) / T}+1} \quad \text { and } \quad \bar{f}_{i}=\frac{1}{e^{\left(\epsilon^{*}+v\right) / T}+1} .
$$

$\epsilon^{*}=\sqrt{k^{2}+M_{N}^{* 2}}$, is the effective nucleon energy, and $v=$ $\mu_{N}-g_{\omega} \omega-I_{3 i} g_{\rho} b_{03}$ is the effective nucleon chemical potential. The term $\Lambda_{v} g_{\omega}^{2} g_{\rho}^{2} b_{03}^{2} \omega_{0}^{2}$ accounts for the $\omega$ - $\rho$ interaction as proposed in [11,12] and already considered in [22].

For the parameters, we used [23] $g_{\sigma}^{q}=5.957, g_{\omega}=8.981$, and $g_{\rho}=8.651$, with $g_{\omega}=3 g_{\omega}^{q}$ and $g_{\rho}=g_{\rho}^{q}$. We have taken the standard values for the meson masses, $m_{\sigma}=550 \mathrm{MeV}$, $m_{\omega}=783 \mathrm{MeV}$, and $m_{\rho}=770 \mathrm{MeV}$. Note that the $s$ quark is unaffected by the $\sigma, \omega$, and $\rho$ mesons, i.e., $g_{\sigma}^{s}=g_{\omega}^{s}=g_{\rho}^{s}=0$. The kaon couplings are given by $g_{\omega K}=\frac{1}{3} g_{\omega}, g_{\rho K}=g_{\rho}$ as in [16].

After a self-consistent calculation, the kaon effective mass $m_{K}^{*}$ can be parametrized as [30]

$$
m_{K}^{*}=m_{K}-g_{\sigma K}(\sigma) \sigma \simeq m_{K}-\frac{1}{3} g_{\sigma}\left(1-\frac{a_{K}}{2} g_{\sigma} \sigma\right) \sigma,
$$

where $a_{k}=0.00045043 \mathrm{MeV}^{-1}$ for $R_{N}=0.6 \mathrm{fm}$. This determines $g_{\sigma K}$ which is a density dependent parameter. 
TABLE I. Symmetry energy, its slope, and related model parameters.

\begin{tabular}{lccclc}
\hline \hline Model & $L(\mathrm{MeV})$ & $\mathcal{E}_{\text {sym }}(\mathrm{MeV})$ & $\rho_{0}\left(\mathrm{fm}^{-3}\right)$ & \multicolumn{1}{c}{$\Lambda_{v}$} & $g_{\rho}$ \\
\hline QMC & 93.5 & 33.70 & 0.15 & 0.0 & 8.8606 \\
QMC & 70.5 & 31.88 & 0.15 & 0.03 & 9.2463 \\
QMC & 59.3 & 30.87 & 0.15 & 0.05 & 9.5335 \\
GM1 & 93.8 & 32.47 & 0.153 & 0.0 & 8.0104 \\
GM1 & 70.8 & 29.57 & 0.153 & 0.03 & 8.5830 \\
GM1 & 59.6 & 27.80 & 0.153 & 0.037 & 8.7358 \\
\hline \hline
\end{tabular}

Finally, the total energy density of the nuclear matter with kaons at finite temperature becomes

$$
\varepsilon=\varepsilon_{B}+\varepsilon_{K},
$$

where [22]

$$
\begin{aligned}
\varepsilon_{B}= & \frac{2}{(2 \pi)^{3}} \sum_{i=p, n} \int d^{3} k\left[\epsilon^{*}\left(f_{i}+\bar{f}_{i}\right)\right] \\
& +\frac{1}{2} m_{\sigma}^{2} \sigma^{2}-\frac{1}{2} m_{\omega}^{2} \omega_{0}^{2}-\frac{1}{2} m_{\rho}^{2} b_{03}^{2} \\
& +g_{\omega} \omega_{0} \rho_{B}+\frac{1}{2} g_{\rho} b_{03} \rho_{3}-\Lambda_{v} g_{\omega}^{2} g_{\rho}^{2} b_{03}^{2} \omega_{0}^{2} .
\end{aligned}
$$

As mentioned in the Introduction, the effect of the slope of the symmetry energy on the kaon condensation is an important quantity to be discussed. We first outline important aspects of the symmetry energy and its slope. The symmetry energy is defined as

$$
\mathcal{E}_{\text {sym }}=\frac{1}{2}\left[\frac{\partial^{2}\left(\varepsilon_{B} / \rho_{B}\right)}{\partial \alpha^{2}}\right]_{\alpha=0}=\frac{k_{F}^{2}}{6 E_{F}}+\frac{g_{\rho}^{2}}{4 m_{\rho}^{* 2}} \rho_{B},
$$

where $\varepsilon_{B}$ is the energy density, $\alpha$ is the asymmetry parameter $\alpha=\left(\rho_{n}-\rho_{p}\right) / \rho_{B}, \rho_{B}=\rho_{n}+\rho_{p}, E_{F}=\sqrt{k_{F}^{2}+M_{0}^{* 2}}$, where $M_{0}^{*}$ is the nucleon effective mass at saturation density and $k_{F}=\left(3 \pi^{2} \rho_{B} / 2\right)^{1 / 3}$,

$$
m_{\rho}^{* 2}=m_{\rho}^{2}+2 \Lambda_{v} g_{\omega}^{2} g_{\rho}^{2} \omega_{0}^{2},
$$

and the slope of the symmetry energy is

$$
L=\left[3 \rho_{B} \frac{\partial \mathcal{E}_{\mathrm{sym}}}{\partial \rho_{B}}\right]_{\rho_{B}=\rho_{0}} .
$$

We consider six different EoS, obtained from the QMC model and the GM1 parametrization of the NLWM. Both models have a similar symmetry energy and corresponding slope at the saturation density. Including the $\omega \rho$ term, we build other EOS from these models by changing the symmetry energy slope at saturation and keeping fixed the symmetry energy as 21.74 MeV at $\rho=0.1 \mathrm{fm}^{-3}$. For each model, QMC and GM1, we have fixed three different values of the slope $L$, roughly the same for both models, namely $L \sim 59.5,70.5,93.5 \mathrm{MeV}$. With a similar behavior of the symmetry energy at saturation, we can discuss how the other properties of the EOS affect the properties of neutron stars containing a kaon condensate. In Table I we display the $L$ values and the related $\Lambda_{v}$ and $g_{\rho}$ for the models we investigate.
In GM1, the coupling to the scalar $\sigma$ field is fixed to a kaon optical potential in symmetric nuclear matter at saturation, $V_{K}=-125 \mathrm{MeV}$, a value suggested by chiral models [31]. For this value of the kaon optical potential we obtain a secondorder phase transition from a pure hadronic phase to a hadronic phase with kaons. A more attractive potential lowers the kaon onset density, and the transition to the hadronic phase is a first-order one [16]. Within the QMC model this quantity is an output, and with the present choice of parameters $V_{K}=$ $-123 \mathrm{MeV}$ [20] at saturation, very close to the above value taken for GM1.

\section{B. Protoneutron star properties}

For neutron stars, their particle composition is determined by the requirements of charge neutrality and $\beta$-equilibrium conditions under the weak processes $n \Rightarrow p+l+\bar{v}$ and $n+l \Rightarrow p+v$, implying that

$$
\mu_{n}=\mu_{p}+\mu_{e}
$$

and

$$
\rho_{e}+\rho_{\mu}+\rho_{K}=\rho_{p}
$$

If neutrino trapping is imposed to the system, the beta equilibrium condition is altered to

$$
\mu_{n}=\mu_{p}+\left(\mu_{e}-\mu_{v_{e}}\right)
$$

In this work, different snapshots of the star evolution are simulated through different entropies per particle and trapped neutrinos. At first, the star is relatively warm (represented by fixed entropy per particle) and has a large number of trapped neutrinos (represented by fixed lepton fraction). As the trapped neutrinos diffuse out, they heat up the star [15]. Finally, the star is considered cold (zero temperature) and deleptonized:

(i) $S=1, Y_{l}=0.4$,

(ii) $S=2, \mu_{v_{l}}=0$,

(iii) $S=0, \mu_{v_{l}}=0$.

The last scenario has already been considered in [20]. We next discuss the first two cases and also the possibility (only for academic purposes) that the temperature is fixed through out the star. The number of muons and muon neutrinos is negligible when the fraction of electrons and electron neutrinos is fixed at 0.4 and, therefore, in this case we do not include muons in the calculation. For the cases without neutrinos, muons are also considered.

\section{RESULTS AND DISCUSSION}

In the following, we discuss the effect of the slope $L$ on the properties of warm stars with a kaon condensate described by two different models, QMC and GM1. We first refer to the influence of $L$ on the EOS, the kaon onset, and the kaon fraction. In a second part the consequences on the star properties, such as radius and mass, and the cooling of protoneutron stars are analyzed. 


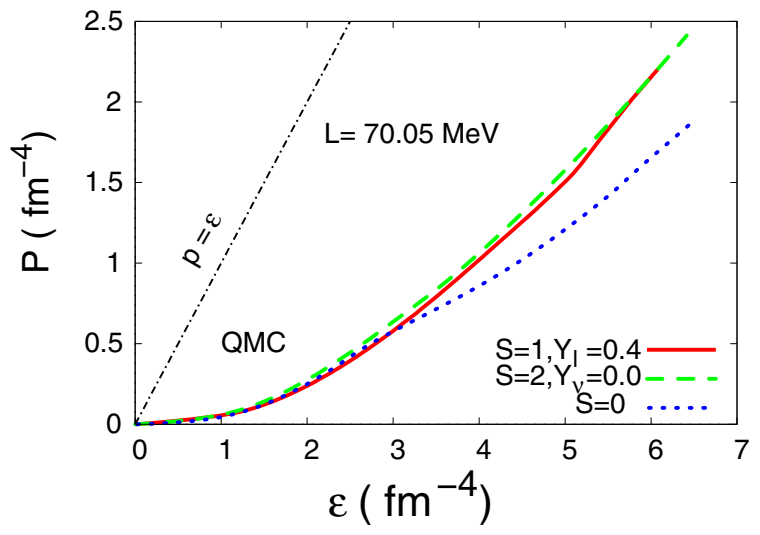

FIG. 1. (Color online) Equation of states for the QMC model and $L=70.05 \mathrm{MeV}$ for three situations of interest in the star evolution.

\section{A. EOS and composition of matter}

In Fig. 1, we plot three curves related to the equations of state obtained with the QMC model and a fixed value of the symmetry energy for three snapshots of the star evolution, which will be discussed next, namely, $S=1$ and trapped neutrinos, $S=2$, and $S=0$ without neutrinos. For the other values of the symmetry energy, the curves present a similar behavior.

At this point, it is important to understand the finite size effects of the quark bags in medium. With this purpose we plot in Fig. 2 the nucleon effective mass and radius as a function of the density for a fixed value of the symmetry energy. One

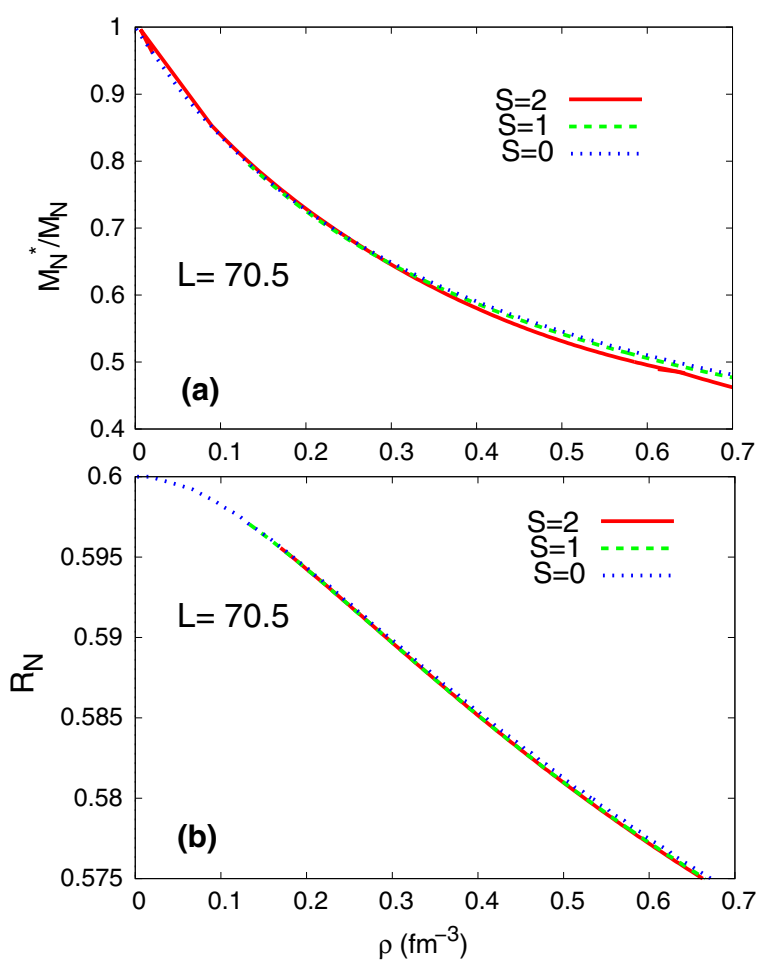

FIG. 2. (Color online) (a) Nucleon effective mass and (b) radius for the QMC model and $L=70.05 \mathrm{MeV}$ for three situations of interest in the star evolution.

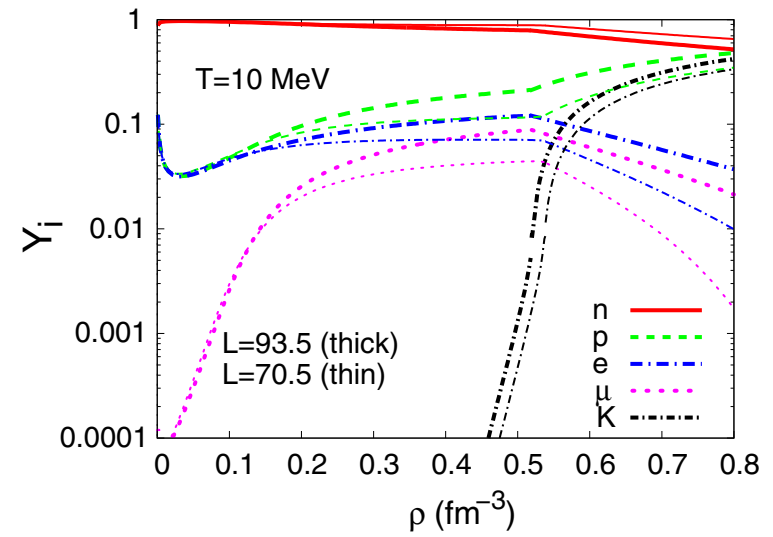

FIG. 3. (Color online) Particle fractions obtained with the QMC model for $T=10 \mathrm{MeV}, L=93.5 \mathrm{MeV}$ (thick line), and $L=70.5 \mathrm{MeV}$ (thin line).

can see that both quantities decrease with the increase of density, the effective mass reducing its value by roughly 50 percent within the range of densities considered. Moreover, at densities attained in stellar matter, the total volume occupied by a nucleon bag varies between $0.90 \mathrm{fm}^{3}$, at low densities, and $0.77 \mathrm{fm}^{3}$, at densities in the center of the star.

In [32] it was shown, for $T=0 \mathrm{MeV}$, that (a) the larger $L$, the larger the fraction of kaons at a given density; (b) the onset of kaon condensation occurs at similar or slightly smaller densities for lower values of $L$. It was also pointed out that the EoS with the lower slope $L$ is softer, giving rise to stars with smaller radii and with a larger total strangeness content because of their larger central densities.

We first discuss the effect of the temperature on the kaon onset. The EOS is calculated at a fixed temperature, even though inside a compact object the temperature is not expected to be constant. In Fig. 3 we plot the particle fraction for a fixed temperature $T=10 \mathrm{MeV}$ and two different $L$ values within the QMC model. The temperature helps the appearance of kaons (strangeness): they appear at a smaller density as compared with the results presented in [20] for a zero temperature system. Similar results were obtained previously [28,33]. Moreover, it is clear that a larger slope enhances the production of kaons. A larger $L$ favors larger proton and electron fractions, and, therefore, we may also expect a larger kaon fraction, since kaons replace the electrons.

After a short initial time, the entropy is practically constant inside the star, and, therefore, in the following we present results obtained for a fixed entropy per baryon, for both matter with trapped neutrinos and $S=1$ and without neutrinos and $S=2$ [15]. In Fig. 4(a), the results for kaon fractions obtained at a fixed entropy per baryon $S=1$ and a lepton fraction of $Y_{l}=0.4$ are shown for the QMC and GM1 models and two different values of $L$. The onset of a condensate of kaons occurs only at a density $\sim 0.15-0.2 \mathrm{fm}^{-3}$ above the onset of thermal kaons. Within GM1, kaons appear at lower densities and, for a given density, in larger amounts than QMC, even when the same slope $L$ is chosen. Hence, stars described by the QMC model present a lower amount of strangeness.This behavior results from a softer EOS at densities above saturation within QMC. 

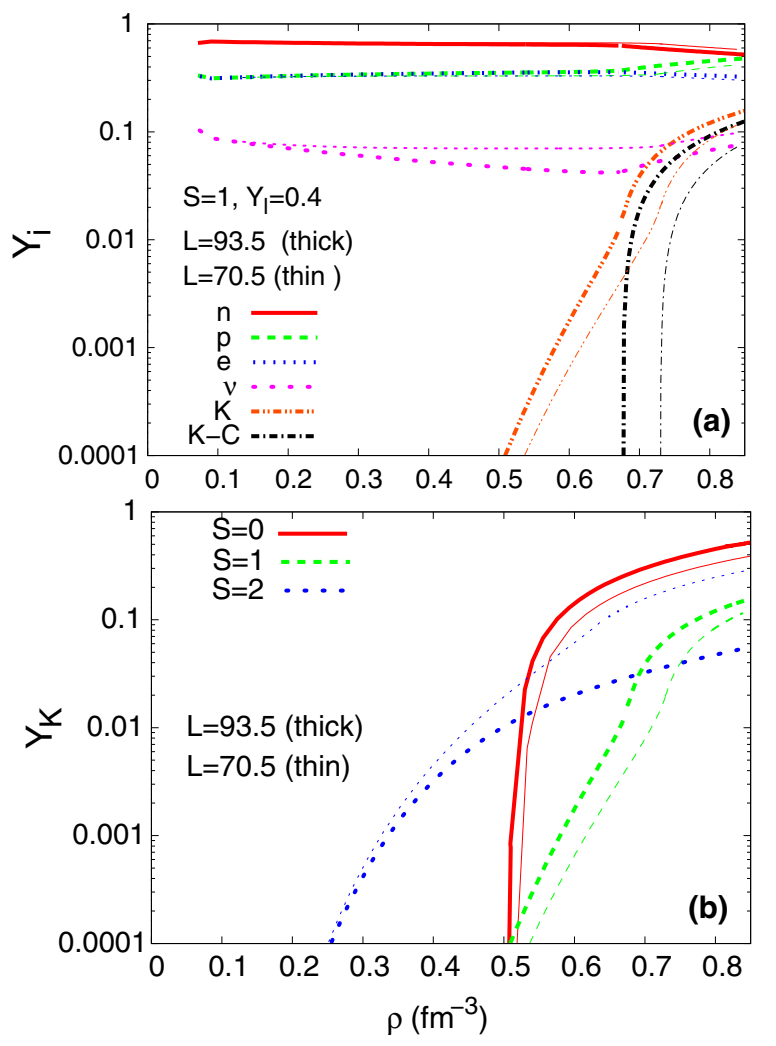

FIG. 4. (Color online) Warm matter with trapped neutrinos, $S=$ 1 and $Y_{l}=0.4$. (a) Particle fractions versus the baryonic density within QMC for two different values of the slope $L$. (b) Kaon fraction for both QMC and GM1.

It is seen that smaller values of $L$ give rise to smaller amounts of strangeness; see Fig. 4(b). This same conclusion was drawn in [22], where hyperons (instead of kaons) were considered. The only exception is $S=2$ : in this case the kaon condensation for $L=93 \mathrm{MeV}$ occurs at a density above the range of densities shown, and, therefore, the kaon fraction is always below the values obtained for $L=70 \mathrm{MeV}$, which predicts a kaon condensate above $0.6 \mathrm{fm}^{-3}$. The presence of neutrinos also disfavors the formation of kaons as seen comparing the $S=1$ curves, obtained with trapped neutrinos, with the ones obtained for $S=0$ and $S=2$ neutrino free matter. The total amount of kaons in the star is ultimately dictated by the central density that is larger for the softer EOS.

In Fig. 5 the density dependence of the kaon effective mass is shown for several values of the entropy per baryon and for different values of the symmetry energy slope. Up to two times the saturation density, the curves are practically identical, i.e., no dependence on the slope and temperature is noticed, but at high densities the mass decreases faster for lower temperatures. In particular, for $S=2$ and $L=93 \mathrm{MeV}$, the mass remains quite high, and consequently no kaon condensate is formed, since a larger mass delays the onset of kaons.

In Fig. 6 we plot the neutrino chemical potential as a function of the baryonic chemical potential for $S=1$ and $Y_{l}=0.4$ for different values of the symmetry energy slope with QMC and GM1 models. Lower values of the slope correspond

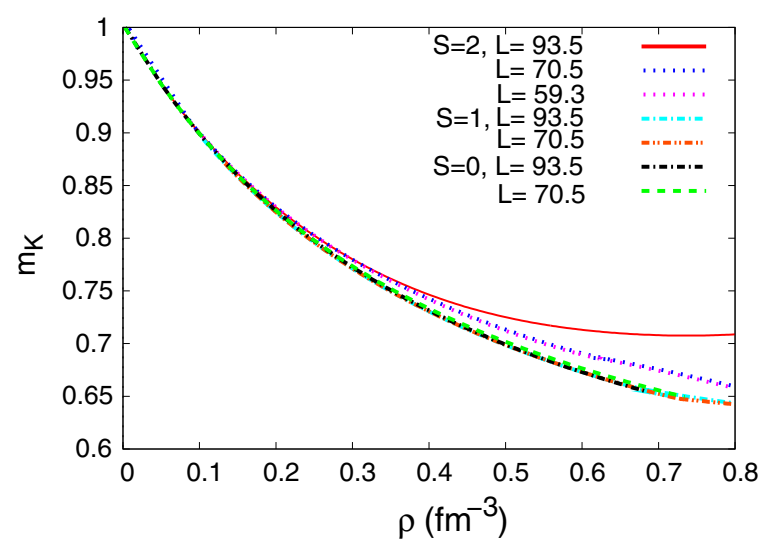

FIG. 5. (Color online) Kaon effective mass versus density in $\beta$ equilibrium matter for different values of the entropy per baryon $S$ and the slope $L$. Matter for $S=1$ contains trapped neutrinos with $Y_{l}=0.4$ and all other curves were obtained for neutrino free matter.

to larger amounts of neutrinos in matter with a fixed fraction of leptons, because a smaller $L$ favors smaller amounts of electrons at large densities.

The kink at a chemical potential above $1300 \mathrm{MeV}$ occurs at the onset of the kaon condensate. After its onset, the number of electrons decreases rapidly due to the charge neutrality condition. For a fixed lepton the neutrino abundance increases to compensate the decrease of the electrons. Comparing the models, it is clear that within GM1 the amount of neutrinos is larger, and, therefore, a larger neutrino chemical potential is obtained. We point out that the kink in the chemical potential due to the onset of the condensate is more pronounced for GM1 and occurs at lower densities. This is due to the onset of a kaon condensation at lower densities and larger amounts of condensed kaons at a given density. Consequently, we may expect that during the star cooling a smaller amount of neutrinos is emitted and the probability of the occurrence of a black hole will be smaller within QMC. We come back to this point later.

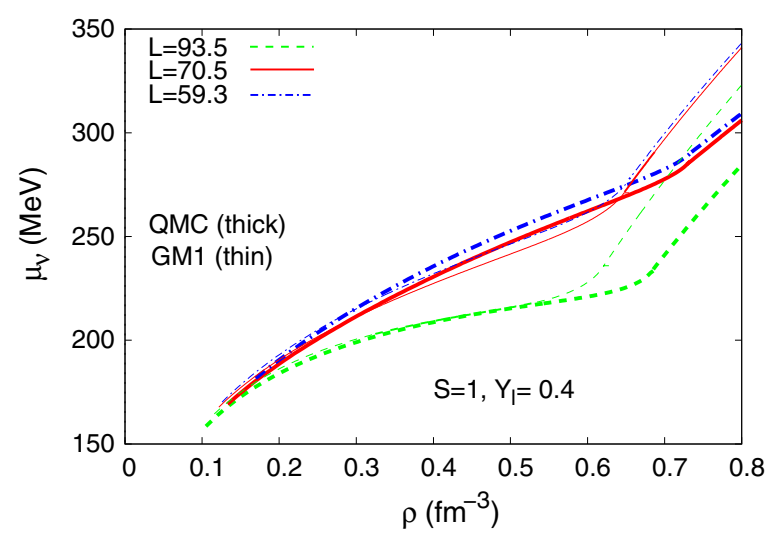

FIG. 6. (Color online) Neutrino chemical potential versus baryon density for $\beta$-equilibrium matter at $S=1$ with the lepton fraction $Y_{l}=0.4$. Results for GM1 and QMC are shown for three different values of $L$. 


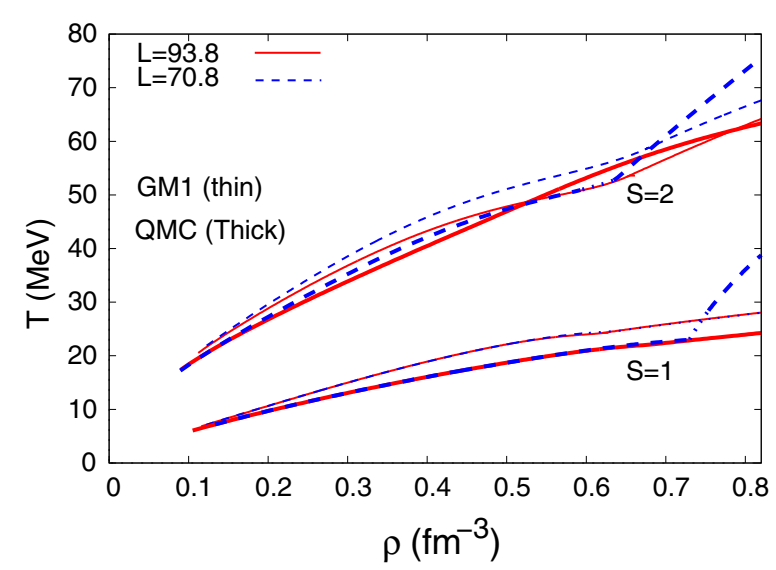

FIG. 7. (Color online) Temperature versus density for neutrino free matter with $S=2$ and matter with neutrino trapped matter with $S=1$.

The temperature of the system is depicted as a function of the baryonic density for $S=2$ neutrino free $\beta$-equilibrium matter and $S=1 \beta$-equilibrium matter with trapped neutrinos in Fig. 7. As compared with the results shown in [21], the system with kaons can reach temperatures inside the star that are much higher than the ones attained when hyperons are included in its core, when the highest temperature is $35 \mathrm{MeV}$. It is the kaon condensation the reason for this behavior. It is seen that, for $S=2, L=93 \mathrm{MeV}$, the temperature does not increase so much because the kaons do not condensate in the range of densities shown.

We also see from Fig. 7 that the temperature values are somewhat similar, but still slightly larger for the smaller $L$ values if $S=2$. In matter with larger values of $L$ the number of neutrons and protons is closer, which corresponds to a smaller temperature if we fix the entropy. No $L$ differences are seen in matter with $S=1$, because in this case the entropy has an important contribution from neutrinos due to their almost zero mass.

\section{B. Kaonic star properties}

Once the EoS are determined, we use them as input in the Tolman-Oppenheimer-Volkov equations to obtain the stellar macroscopic properties, which are shown in Table II and Figs. 8 and 9. The result for $T=10 \mathrm{MeV}$ is added only for completeness.

For the present choice of the QMC parameters a baryon bag occupies a volume that varies between $v \sim 0.9 \mathrm{fm}^{3}$ at $\rho=0$ and $v \sim 0.77 \mathrm{fm}^{3}$ at $\rho \sim 0.9 \mathrm{fm}^{-3}$ independently of the temperature. This volume is still compatible with no bag overlapping at the central densities obtained for the maximum mass configurations. For $T=0$, the star with the largest central density has $\rho_{c}=0.88 \mathrm{fm}^{-3}$, so that the volume occupied by one particle is $1 / 0.88=1.14 \mathrm{fm}^{3}$. As close packing of equal spheres fills $74 \%$ of the total volume, one particle would have an available volume of $v \sim 0.74 \times 1.14=0.84 \mathrm{fm}^{3}$, larger than the volume that is really occupied by a bag at those densities $\left(0.77 \mathrm{fm}^{3}\right)$.

The maximum masses for the cases with fixed entropy do not show a clear behavior with the slope, but for a stable zero temperature star they tend to decrease with the decrease of the slope for both models. If the slope $L$ is small the trend may invert due to two competing effects: (a) a smaller slope $L$ means a softer EOS and, therefore, smaller maximum masses; (b) however, the onset of kaons for a softer EOS occurs at larger densities, as seen in Table II from the central energy densities of the threshold stars for the kaon onset, and, as a result, stars

TABLE II. Star properties for the EOS described in the text for QMC and GM1 models and the three $L$ values: the maximum gravitational and baryonic masses, corresponding radius, the central energy density, onset energy density of the kaon condensate, the mass of a star with this threshold mass, and radii of $1.4 M_{\odot}$ star.

\begin{tabular}{|c|c|c|c|c|c|c|c|c|c|}
\hline Model & Type & $L(\mathrm{MeV})$ & $M_{\max }\left(M_{\odot}\right)$ & $M_{b \max }\left(M_{\odot}\right)$ & $R(\mathrm{~km})$ & $\varepsilon\left(\mathrm{fm}^{-4}\right)$ & $\varepsilon^{K}\left(\mathrm{fm}^{-4}\right)$ & $M_{\max }^{K}\left(M_{\odot}\right)$ & $R\left(1.4 M_{\odot}\right)(\mathrm{km})$ \\
\hline QMC & $T=10 \mathrm{MeV}$ & 93.5 & 2.03 & 2.37 & 12.8 & 5.66 & 2.80 & 1.86 & \\
\hline QMC & $S=1, Y_{l}=0.4$ & 93.5 & 2.03 & 2.25 & 11.7 & 5.26 & 4.09 & 1.99 & 13.12 \\
\hline QMC & $S=1, Y_{l}=0.4$ & 59.03 & 2.04 & 2.25 & 10.9 & 5.97 & 4.30 & 1.97 & 11.91 \\
\hline QMC & $S=2$ & 93.5 & 2.51 & 3.11 & 12.2 & 5.35 & 3.03 & 2.30 & 14.15 \\
\hline QMC & $S=2$ & 70.5 & 2.15 & 2.51 & 11.8 & 5.86 & 3.44 & 2.04 & 13.69 \\
\hline QMC & $S=0$ & 93.5 & 1.98 & 2.25 & 12.08 & 5.41 & 2.93 & 1.86 & 13.58 \\
\hline QMC & $S=0$ & 70.5 & 1.94 & 2.12 & 11.85 & 5.61 & 2.96 & 1.81 & 13.19 \\
\hline QMC & $S=0$ & 59.03 & 1.95 & 2.18 & 11.7 & 5.75 & 3.07 & 1.82 & 13.06 \\
\hline GM1 & $S=1, Y_{l}=0.4$ & 93.8 & 2.24 & 2.52 & 11.9 & 5.52 & 3.70 & 2.17 & 13.13 \\
\hline GM1 & $S=1, Y_{l}=0.4$ & 70.8 & 2.23 & 2.61 & 11.7 & 5.64 & 3.91 & 2.18 & 12.96 \\
\hline GM1 & $S=1, Y_{l}=0.4$ & 59.6 & 2.24 & 2.53 & 11.7 & 5.64 & 3.92 & 2.18 & 12.88 \\
\hline GM1 & $S=0$ & 93.8 & 2.14 & 2.46 & 12.8 & 4.63 & 2.70 & 2.03 & 13.82 \\
\hline GM1 & $S=0$ & 70.8 & 2.06 & 2.39 & 12.4 & 4.86 & 2.94 & 2.02 & 13.23 \\
\hline GM1 & $S=0$ & 59.6 & 2.06 & 2.39 & 12.3 & 4.96 & 2.96 & 1.95 & 13.06 \\
\hline
\end{tabular}



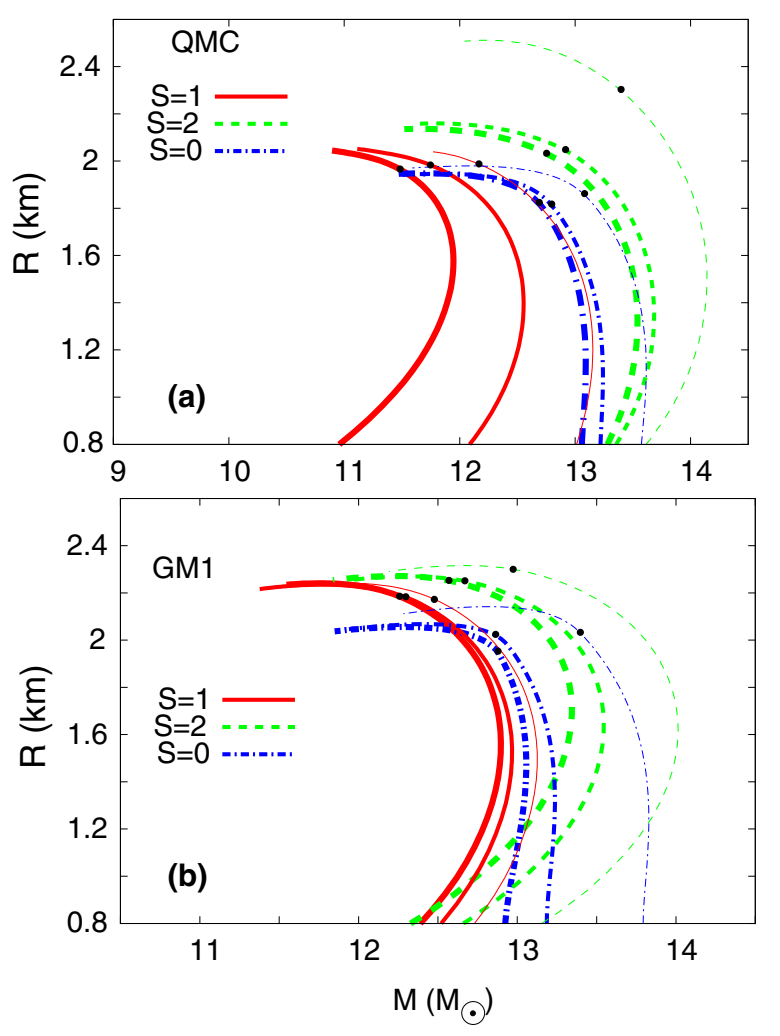

FIG. 8. (Color online) Mass-radius profiles for stars with $S=1$ and fixed lepton fraction $Y_{l}=0.4$ (red full lines), $S=2$ and neutrino free matter (green dashed lines), and cold matter (blue dotted lines), for different values of the symmetry energy slope: $L=59$ (thick), 70 (medium), and 93 (thin) MeV, with (a) QMC and (b) GM1. The black dots indicate the onset of kaon condensate.

obtained from an EOS with larger $L$ contain larger amounts of kaons, which soften the EOS. Therefore, decreasing $L$ reduces the maximum star mass until a critical value of $L$ when the onset of kaons is shifted to much larger densities so that the overall softening due to the kaon fraction becomes negligible. $L=$ $59 \mathrm{MeV}$ is one of these critical values of the slope. This effect is present in both GM1 and QMC and for cold and warm stars.

A comment should also be made concerning the differences of maximum masses with $L$. Within the QMC the maximum mass diference is quite small, in fact not larger than $\sim 0.04 M_{\odot}$ except for the $S=2, L=93 \mathrm{MeV}$ case, when the much larger mass is due to the nonexisting kaon condensation in the star, since only thermal kaons are present. Within GM1 the differences are larger, but the main considerations above remain valid. The difference in this case is the fact that the nucleonic GM1 EOS is harder than QMC at intermediate densities.

The star radii, on the other hand, decrease with the decrease of the slope, see Fig. 8, as already seen in $[11,14]$ for different parametrizations of the NLWM (excluding IU-FSU). In these figures the black dots indicate the onset of thermal kaons, which occurs for quite massive stars. Therefore, we may state that the radius difference between the families of stars is mainly due to the different behavior of the symmetry energy with density and not to the presence of the kaons. According to [34], the radii of canonical $1.4 M_{\odot}$ neutron stars should lie within the range 9.7-13.9 km. In Table II we show our results
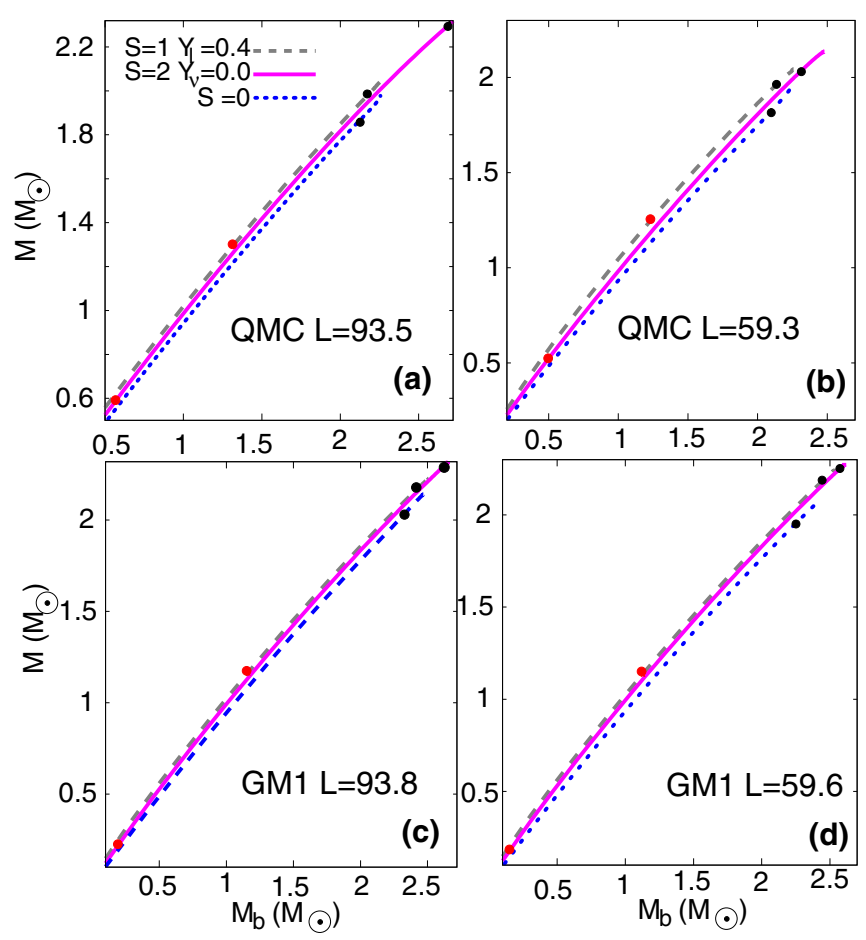

FIG. 9. (Color online) Baryonic versus gravitacional masses for stars with $S=1$ and fixed lepton fraction $Y_{l}=0.4$ (top dashed curve), $S=2$ and neutrino free matter (middle full curve), and cold matter (bottom dotted curve), for (a) and (c) $L=93.5 \mathrm{MeV}$ and (b) and (d) $L=59.3 \mathrm{MeV}$. The results were obtained with (a) and (b) QMC and (c) and (d) GM1. The black full dots indicate the minimal mass configuration which contains a kaon condensate in the core, and the red asterisks the minimal configuration with a fraction $10^{-8}$ or larger of thermal kaons.

for these canonical stars and see that, except for the $S=2, L=$ 93.5/93.8 case, a star which is not stable, our results fall inside the expected range. On the other hand, other two different analyses of five quiescent low-mass $\mathrm{x}$-ray binaries in globular clusters were performed to establish possible neutron star radii ranges. In the first analysis [35], all neutron stars were assumed to have the same radii in the range $R=9.1_{-1.5}^{+1,3}$. The second calculation [36], based on a Bayesian analysis, estimates that neutron stars radii should lie in between 10.9 and $12.7 \mathrm{Km}$. It is important to have in mind that measurement and assessment of neutron star radii still remain to be better understood, but if the above mentioned constraints are to be validated, our results would only be in accordance with the second analysis.

The kaon onset energy density increases with decreasing $L$ as already referred; see $\epsilon^{K}$ in Table II. However, since the existence of kaons softens the EOS, the kaon threshold star mass is generally smaller for the smaller $L$. Notice that both models, even with the inclusion of kaons and the $\omega$ - $\rho$ interaction, known to soften the EoS, may account for the description of very massive stars.

In Fig. 9 we display, both for the QMC and GM1 models and two values of the symmetry energy slope, the baryonic versus gravitational masses for stars at different snapshots of their lives [37]: (a) immediately after the core bounce with trapped neutrinos $S=1$ and fixed lepton fraction $Y_{l}=0.4$; 
(b) after neutrino diffusion $Y_{v}=0$ and core heating due to deleptonization with $S=2$ (the maximum entropy per baryon $S=2$ is attained at $t=15 \mathrm{~s}$ ); (c) after core cooling with $S=0$ and $Y_{v}=0$. If no accretion occurs during the cooling process. the transition between the different stages occurs at constant baryonic mass, i.e., along vertical lines [15]. We identify, with a full black dot and a red asterisk, respectively, the minimal configuration with a kaon condensate in the center and with a fraction of kaons equal to $10^{-8}$.

Some configurations obtained for $S=2$ and neutrino-free matter cannot be populated since their baryonic mass is larger than the maximum baryonic mass obtained with $S=1$ and trapped neutrinos. In particular, all stars with $M>2.05 M_{\odot}$ for $S=2$ and $L=93 \mathrm{MeV}$ belong to this set of stars. It is also seen that within the QMC model (but not GM1) it may occur that a star has a kaon condensate in its center during the neutrino trapped phase. After the neutrinos diffuse out the condensate melts and finally it is formed once more after cooling. These transformations should result in neutrino signals after the supernova explosion and before the cooling of the star.

Some conclusions are in order: (a) From both Fig. 8 and Table II we conclude that for $L=93 \mathrm{MeV}$ no black hole will be formed during the cooling process since the maximum baryonic mass at $S=1$ and $Y_{l}=0.4$ is not larger than the maximum baryonic mass at $S=0$. This is not the case for GM1. This model predicts the formation of low mass back holes during cooling. (b) Decreasing the symmetry energy slope $L$ may modify some of the above conclusions. In particular, for $L=70 \mathrm{MeV}$ (but not anymore for $59 \mathrm{MeV}$ ) within QMC there is a small range of star configurations $\left(\Delta M \sim 0.07 M_{\odot}\right)$ that will decay to a black hole during cooling. Within GM1 the number of star configurations that decay into black holes increases when $L$ goes from 93 to $70 \mathrm{MeV}$, but decreases if $L$ is further reduced to $59 \mathrm{MeV}$. This is due the smaller kaon content in these last stars together with a central density that is not much larger. (c) In same cases the cooling of the stars that contain a kaon condensate involves the melting of the condensate at an intermediate stage $(S=2)$ and a second formation of the condensate at $T=0$.

We should point out that we are studying the evolution of stars without considering finite size effects as in [38]. In the present calculation the kaon potential is not strong enough to give rise to a first-order phase transition, and corresponds to a second-order phase transition.

\section{CONCLUSIONS}

In the present work we have revisited the QMC model at finite temperature to investigate the thermal kaon effects on stellar properties. The $\omega$ - $\rho$ interaction was included because it softens the very hard QMC symmetry energy at high densities and can be used to tune the values of the slope of the symmetry energy. As had already been seen in [14,22], lower values of the slope yield smaller amounts of strangeness if hyperons are considered for a given density. The same conclusion is here obtained if kaons are the carriers of strangeness instead of the hyperons.

As compared with the results obtained with the GM1 parametrization of the NLWM, the QMC EoS is generally softer, the only exception being the $S=2$ case for $L=$ 93.5 $\mathrm{MeV}$ (see Table II), which is due to the fact that no kaon condensate is formed because the central temperatures of the star lie above the melting temperature of the condensate. A softer EOS at intermediate densities implies a smaller amount of kaons, and as a consequence within QMC no black hole formation is expected during the cooling of the a protoneutron star with a kaon condensate in the core, if $L$ is large enough. For smaller values of $L$, but not too small, the set of stars that could cool to a black hole is very reduced and certainly much smaller than what is expected with GM1.

It is interesting to identify the role of the density dependence of the symmetry energy on the possible evolution of a compact star with a kaon condensate. Within QMC no black hole is formed either if $L$ is large or $L$ small. This is due to the balance between the softening of the EOS when $L$ is smaller, together with a less pronounced softening of the EOS because fewer kaons are formed. Within GM1 there are always a quite large set of stars that cool to a black-hole, although this set is larger for intermediate values of $L$.

We have also shown that the complex evolution of the star may include the melting and formation of a new kaon condensate. The first transformation is driven by the neutrino diffusion and the second is due to cooling. These processes could be responsible for a neutrino signal followed by a gamma ray burst after the supernova explosion.

Finally, we point out that both models can describe very massive stars, namely stars as massive as the pulsars PSR J16142230 [7] and PSR J0348+0432 [8].

\section{ACKNOWLEDGMENTS}

This work was partially supported by the initiative QREN financed by the UE/FEDER through the Programme COMPETE under the project PTDC/FIS/113292/2009, by CNPq (Brazil) and FAPESC (Brazil) under project 2716/2012,TR 2012000344, and by NEW COMPSTAR, a COST initiative. P.K.P. acknowledges the warm hospitality at both the University of Coimbra and Universidade Federal de Santa Catarina and D.P.M. at the Universidad de Alicante, where parts of this work were carried out.
[1] B. G. Todd-Rutel and J. Piekarewicz, Phys. Rev. Lett. 95, 122501 (2005); F. J. Fattoyev and J. Piekarewicz, Phys. Rev. C 82, 025805 (2010).

[2] G. A. Lalazissis, J. Konig, and P. Ring, Phys. Rev. C 55, 540 (1997).
[3] J. Piekarewicz, Phys. Rev. C 66, 034305 (2002); 69, 041301 (2004).

[4] S. S. Avancini, J. R. Marinelli, D. P. Menezes, M. M. W. Moraes, and A. S. Schneider, Phys. Rev. C 76, 064318 (2007); S. S. Avancini, J. R. Marinelli, D. P. Menezes, 
M. M. W. Moraes, and C. Providência, ibid. 75, 055805 (2007).

[5] J. Cottam, F. Paerels, and M. Mendez, Nature (London) 420, 51 (2002).

[6] D. Sanwal, G. G. Pavlov, V. E. Zavlin, and M. A. Teter, Astrophys. J 574, L 61 (2002).

[7] P. Demorest, T. Pennucci, S. Ransom, M. Roberts, and J. Hessels, Nature (London) 467, 1081 (2010).

[8] J. Antoniadis et al., Science 340, 6131 (2013).

[9] J. M. Lattimer and Y. Lim, Astrophys. J. 771, 51 (2013).

[10] M. B. Tsang et al., Phys. Rev. C 86, 015803 (2012); W. G. Newton, M. Gearheart, J. Hooker, and B.-A. Li, in Neutron Star Crust, edited by C. A. Bertulani and J. Piekarewicz (Nova Science, Hauppauge, NY, 2012); I. Vidaña, C. Providência, A. Polls, and A. Rios, Phys. Rev. C 80, 045806 (2009); C. Ducoin, J. Margueron, C. Providência, and I. Vidaña, ibid. 83, 045810 (2011).

[11] C. J. Horowitz and J. Piekarewicz, Phys. Rev. Lett. 86, 5647 (2001); J. K. Bunta and Š. Gmuca, Phys. Rev. C 68, 054318 (2003).

[12] F. J. Fattoyev, C. J. Horowitz, J. Piekarewicz, and G. Shen, Phys. Rev. C 82, 055803 (2010).

[13] C. Providência et al., Eur. Phys. J. A 50, 44 (2014).

[14] R. Cavagnoli, D. P. Menezes, and C. Providência, Phys. Rev. C 84, 065810 (2011).

[15] M. Prakash, I. Bombaci, M. Prakash, P. J. Ellis, J. M. Lattimer, and R. Knorren, Phys. Rep. 280, 1 (1997).

[16] N. K. Glendenning, Compact Stars (Springer-Verlag, New York, 2000).

[17] D. B. Kaplan and A. E. Nelson, Phys. Lett. B 175, 57 (1986); 179, 409(E) (1986)

[18] G. E. Brown, K. Kubodera, M. Rho, and V. Thorsson, Phys. Lett. B 291, 355 (1992).

[19] J. Schaffner and I. N. Mishustin, Phys. Rev. C 53, 1416 (1996).

[20] D. P. Menezes, P. K. Panda, and C. Providência, Phys. Rev. C 72, 035802 (2005).
[21] P. K. Panda, C. Providência, and D. P. Menezes, Phys. Rev. C 82, 045801 (2010).

[22] P. K. Panda, A. M. S. Santos, D. P. Menezes, and C. Providência, Phys. Rev. C 85, 055802 (2012).

[23] P. K. Panda, D. P. Menezes, and C. Providência, Phys. Rev. C 69, 025207 (2004).

[24] N. Gupta and P. Arumugam, Phys. Rev. C 85, 015804 (2012).

[25] A. Mishra, A. Kumar, S. Sanyal, V. Dexheimer, and S. Schramm, Eur. Phys. J. A 45, 169 (2010).

[26] P. A. M. Guichon, Phys. Lett. B 200, 235 (1988); K. Saito and A. W. Thomas, ibid. 327, 9 (1994); P. K. Panda, A. Mishra, J. M. Eisenberg, and W. Greiner, Phys. Rev. C 56, 3134 (1997).

[27] N. Gupta and P. Arumugam, Phys. Rev. C 87, 045802 (2013).

[28] J. A. Pons, J. A. Miralles, M. Prakash, and J. M. Lattimer, Astrophys. J. 553, 382 (2001).

[29] A. Chodos, R. L. Jaffe, K. Johnson, C. B. Thorne, and V. F. Weisskopf, Phys. Rev. D 9, 3471 (1974).

[30] K. Tsushima, K. Saito, A. W. Thomas, and S. V. Wright, Phys. Lett. B 429, 239 (1998).

[31] T. Waas and W. Weise, Nucl. Phys. A 625, 287 (1997); W. Weise and R. Hartle, ibid. 835, 51 (2010).

[32] C. Providência et al., J. Phys.: Conf. Ser. 413, 012023 (2013).

[33] S. Banik, R. Nandi, and D. Bandyopadhyay, Phys. Rev. C 86, 045803 (2012); S. Banik, W. Greiner, and D. Bandyopadhyay, ibid. 78, 065804 (2008).

[34] K. Hebeler, J. M. Lattimer, C. J. Pethick, and A. Schwenk, Phys. Rev. Lett. 105, 161102 (2010).

[35] S. Guillot, M. Servillat, N. A. Webb, and R. E. Rutledge, Astrophys. J. 772, 7 (2013).

[36] J. M. Lattimer and A. W. Steiner, Astrophys. J. 784, 123 (2014).

[37] M. Prakash, J. M. Lattimer, J. A. Pons, A. W. Steiner, and S. Reddy, in Physics of Neutron Star Interiors, edited by D. Blaschke, A. Sedrakian, and N. K. Glendenning, Lecture Notes in Phys. Vol. 578 (Springer, Berlin, 2001), p. 364.

[38] T. Maruyama, T. Tatsumi, D. N. Voskresensky, T. Tanigawa, T. Endo, and S. Chiba, Phys. Rev. C 73, 035802 (2006). 